\title{
A Symmetric RZ-DPSK Based Colorless NG-PON using Optical Carrier Suppression Scheme
}

\author{
AFTAB HUSSAIN*, ABDUL LATIF MEMON**, AND ABDUL QADIR ANSARI*** \\ RECEIVED ON 21.03.2016 ACCEPTED ON 11.05.2016
}

\begin{abstract}
In this paper a simultaneous transmission of a $10 \mathrm{Gbps}$ RZ-DPSK data signal for downstream as well as for upstream is proposed and successfully simulated. An OCS (Optical Carrier Suppression) scheme for generation of second order dual side-band optical carrier is utilized by quadrupling a $10 \mathrm{GHz}$ clockfrequency with a $10 \mathrm{GHz} \mathrm{LN-MZM} \mathrm{(Lithium-Niobate} \mathrm{Mach-Zehnder-Modulator).} \mathrm{The} \mathrm{upper} \mathrm{side} \mathrm{second}$ order band is used to generate a RZ-DPSK (Return to Zero-Differential Phase Shift Keying) data signal at the OLT (Optical Line Terminal). At the receiving ONU (Optical Network Unit) $50 \mathrm{~km}$ away from the OLT the unmodulated lower side second order band coupled with the downlink transmitted signal is utilized for the uplink modulation of $10 \mathrm{Gbps}$ data in RZ-DPSK format. The simulation results show that the performance of the single-tone RZ-DPSK data modulation format is a suitable choice for the WDMPON (Wavelength Division Multiplexing-Passive Optical Network) link with a transmission span of 50 $\mathrm{km}$. The proposed architecture eliminates the need of any pulse carver and mid-span power amplifiers along with the requirement of any power splitting device used in the ONU for colorless uplink transmission. In this scheme, high data rate transmission over long distance is achieved. This scheme merges the boundaries of local access networks and MAN (Metropolitan Area Networks). The proposed scheme is a highly robust, cost effective, backward compatible as well as future proof WDM-PON architecture.
\end{abstract}

Key Words: Long Reach Passive Optical Networks, Return to Zero Differential Phase Shift Keying, Optical Carrier Suppression, Lithium Niobate Mach-Zehnder Modulator.

\section{INTRODUCTION}

ML (Chirped Managed Laser) based RZ-DPSK transmitter designs are efficient and cheap in

the downlink transmission. But performance of remodulation of such signals are limited by the uplink based IM (Intensity Modulation) format against fiber nonlinearities thus restricting the uplink reach of CML based WDM-PON to only $20 / 25 \mathrm{~km}$ distance with high data rates $(\geq 10 \mathrm{Gbps})$. There is therefore, a need for new transmission scheme for uplink, in which the uplink transmission distance is not limited by higher data rates

\footnotetext{
* Department of Electrical Engineering University of Azad Jammu \& Kashmir, Muzaffarabad.

** Department of Telecommunication Engineering, Mehran University of Engineering \& Technology, Jamshoro.

*** Department of Biomedical Engineering, Mehran University of Engineering \& Technology, Jamshoro
}

Mehran University Research Journal of Engineering \& Technology, Volume 36, No. 1, January, 2017 [p-ISSN: 0254-7821, e-ISSN: 2413-7219] 
due to fiber nonlinearities. The new technique should be colorless but without implementing remodulation approach, because the remodulation approach is data as well as distance limited and mainly affected by RBS (Rayleigh Back Scattering) phenomenon.

LR-PONs (Long Reach Passive Optical Networks) are considered a better alternative for FTTx systems deployment in a green-field scenario for integration of wired and wireless services [1-2]. The LR-PON approach integrates the metro and access networks into one single platform, which significantly alleviates operational and capital expenditure. Moreover, such mechanism implements minimization of the number of central offices in low population density areas and enhances power savings, due to reduced number of network nodes and other interconnecting interfaces [3].

Using optical amplification in a WDM environment can enhance the coverage reach from the standard $20 \mathrm{~km}$ to beyond $60 \mathrm{~km}$ while maintaining a 1:32 or higher split ratio (in case of WDM-TDM-PON). But such development regarding greater split ratio and extended reach are also associated with higher insertion losses [4]. In uplink direction, an optical amplifier has to offer a wide dynamic range and fast response time while alleviating waveform distortion i.e. optical surge. In the case of downlink an optical amplifier has to suppress waveform distortions, such as XGM (Cross Gain Modulation) and pattern effects.

EDFAs (Eribium Doped Fiber Amplifier), one of the widely used optical amplifiers, can only function in the C- and Lbands to provide the outstanding power gain and noise performance. The wavelength band and relatively slow speed in fine-tuning the EDFA gain, makes this option unfavorable to the bursty nature of upstream TDMA (Time Division Multiple Access) traffic. Moreover, huge size, external power requirement, exaggerated cross talk, gain saturation and ASE (Amplified Spontaneous Emission) are some major issues associated with EDFA.
SOAs (Semiconductor Optical Amplifiers) on the other hand, have several applications and benefits from the fact that they can function at any wavelength of interest including the $\mathrm{O}$ band along with improved gain dynamics than the EDFA, as established in [5-6]. The high level of noise and inability to provide simultaneous amplification across multiple channels make them to be rarely used as in-line amplifiers. The low production cost, wide bandwidth, small insertion loss as well as fast time response enable them as a potential candidate to be used as an electro-optic transceiver at the user premises in photonic access network.

DRAs (Distributed Raman Amplifiers) execute seamless band-separated, bidirectional amplification due to simple configuration, but their fundamental drawback is the incidence of high-power pump beams inside the feeder fiber that could lead to significant eye safety issues. Therefore, highly sensitive detection modules must be acquired for rapid shutdown of amplifiers and protection switching upon fiber failure detection [7].

Moreover, for the amplification of optical wavelength bands in a full duplex transmission, the mid-span bidirectional optical amplifiers must be constructed to have a band separated configuration with two independent amplifiers for amplification of each downlink as well as uplink. The deployment of any such type of optical amplifiers along the feeder fiber or in the ONU stage for any reason converts the network from passive to active and enhances the network cost.

Another major task for the wide exploitation of WDMPON is the need of a cost-effective wavelength specific light source for each ONU. Among many proposed techniques so far, the centralized light source scheme appears to be the most promising with low cost for the upstream transmission [8]. Carrier distribution [9-10] and downstream wavelength re-modulation [11] are types of centralized light source scheme. The carrier distribution 
scheme is based on provision of two individual wavelengths for each ONU, one for the downlink and the other for the uplink. Hence provision of wavelength management is the major issue of concern. In the downstream wavelength re-modulation technique, the downlink carrier can be modulated at either baseband or subcarriers producing a colorless wavelength independent ONUs. It is a cost effective scheme and required only one wavelength channel per ONU as no seed light is required from OLT for uplink.

DPSK modulation format with balance detection is greatly advantageous in high speed transmission systems due to its superior performance against nonlinear effects, increased receiver sensitivity and enhanced tolerance to coherent crosstalk [12]. Similarly, return to zero coding format offers high receiver sensitivity and high immunity to inter-symbol interferences compared to non-returnto-zero format. Therefore, RZ-DPSK modulation format is the ideal choice for high speed long reach access transmissions system. Orthogonal downstream (DPSK) and upstream (IM) modulation format are used to protect the uplink performance deterioration. In addition to the $3 \mathrm{~dB}$ optical splitter used at the ONU to split apart the downlink signal is a major limiting factor for restricting the uplink transmission reach.

In this article, a novel, highly cost effective, and reduced circuitry based WDM-PON (Wavelength Division Multiplexing Passive Optical Network) having a transmission span of $50 \mathrm{~km}$ is proposed without using any amplification or dispersion compensation devices for existing and future optical wired and WAN (Wireless Access Networks). This novel approach implements the realization of simultaneous transmission of RZ-DPSK modulation format both for downlink as well as for uplink with reduced wavelength management concerns and the elimination of $3 \mathrm{~dB}$ optical splitter at the ONU for extended uplink reach. This is the first colorless WDM-PON demonstration for symmetric $10 \mathrm{Gbps}$ data rate that requires no amplification device at any stage of transmission up to $50 \mathrm{~km}$ over a single, feeder fiber architecture. Similarly, it is the first colorless WDM-PON scheme that utilizes simultaneous RZ-DPSK modulation scheme for both downlink and uplink. Moreover, the transmitter design for both uplink and down link includes only one Mach Zehnder Modulator, without requiring any pulse culver and eliminates the need of optical power splitter at the ONU.

\section{DOWNLINK AND UPLINK CARRIER GENERATION USING OCS APPROACH}

An OCS modulation technique is considered to be an ideal choice for transmission of DWDM (Dense Wavelength Division Multiplexing) channels without any power saturation or other nonlinear impairments with negligible power penalty over extended reach [1315]. For efficient and low cost OCS modulation generation, a lightwave expressed as $\mathrm{E}_{(\mathrm{t})}=\mathrm{E}_{0}{ }^{\mathrm{ej} \omega \mathrm{t}}$ generated from a narrow linewidth laser source, been passed through a dual-arm $\mathrm{LiNbO}_{3}$ modulator (LN-MZM) biased at Null point $\left(V_{\pi}\right)$ and driven in a push-pull format with two opposite polarity $10-\mathrm{GHz}$ clock signals \pm $\mathrm{V}_{\mathrm{RF}} \cos \omega_{\mathrm{m}} \mathrm{t}$ with a frequency of $\mathrm{f}_{\mathrm{m}}=\omega_{\mathrm{m}} / 2 \pi$, could produce a dual tone OCS lightwave signal consisting of two second-order sidebands by quadrupling the RF LO. While the frequency separation of the generated dual tone optical signal is $40 \mathrm{GHz}$. The above phenomenon can be expressed mathematically as [16]:

$$
\begin{aligned}
& E(t)=\frac{1}{2} \alpha E_{0} e^{j \omega_{c} t}\left(e^{\frac{j \pi V_{R F} \cos \omega_{m} t}{V_{\pi}}}+e^{\frac{j \pi V_{R F} \cos \omega_{m} t}{V_{\pi}}}\right) \\
& \approx \alpha E_{0} e^{j \omega_{c} t}\left[-J_{ \pm 2}\left(m_{h}\right) e^{j\left(\omega_{c} \pm 2 \omega_{m}\right) t}+J_{0}\left(m_{h}\right) e^{j \omega_{c} t}\right]
\end{aligned}
$$


In the Equation (2) " $\alpha$ ” represents the insertion loss of the modulator and " $m_{h}$ " denotes the modulation index with a value $=2.405$, defined as:

$m_{h}=\frac{\pi V_{R F}}{V_{\pi}}$

From the Equation (2), it can be seen clearly that the optical carrier and the odd numbered sidebands are suppressed completely and the output OCS signal can be formulated as:

$$
E(t)=-\alpha E_{0} J_{2}(2.405)\left\{e^{j\left(\omega_{c}-2 \omega_{m}\right) t}+e^{j\left(\omega_{c}+2 \omega_{m}\right) t}\right\}
$$

The higher order second-order sideband thus generated is separated by using an optical filter and subjected to be Phase modulation with a 10 -Gbps downlink data using RZ-DPSK modulation technique and then combined with the left over lower order side second-order sideband. The combined signal is sent to the ONU where an interleaver is used to separate both the side bands. The un-modulated lower order side band is used as a carrier for the uplink direction. The next section will elaborate the transmitter design used to modulate the downlink and uplink data signal over the OCS generated side bands.

\section{TRANSMITTER DESIGN AND WORKING PRINCIPLE}

The drive circuitry includes a clock signal generator AND gates, inverters, power combiner and a LN-MZM driven by light source as shown in Fig. 2. A differentially encoded binary data signal of $10 \mathrm{Gbps}$ is combined with a sinusoidal clock signal of $10 \mathrm{GHz}$ using a high speed AND gate to produce a two level positive electronic RZ pulse shape for each "1" bit, and zero pulse for each " 0 " bit as shown in the Inset-I of Fig. 1. Similarly inverted or inverse of the input data signal along with a copy of the sinusoidal clock signal $(10 \mathrm{GHz})$ are combined together using a second high speed AND gate. The output thus produced is inverted to generate a negative electronic RZ pulse shape for each "0" bit and zero pulse for each " 1 " bit respectively as shown in the Inset-II of Fig. 2.

Both outputs thus produced are then combined together using a power combiner to generate a 3-level RZ pulse signal as shown in the Inset-III of Fig. 2.

The 3-levels indicate the three voltage states of the generated RF drive signal i.e. $+1,-1$ normalized at the centre of each information bit, while the " 0 -voltage" state

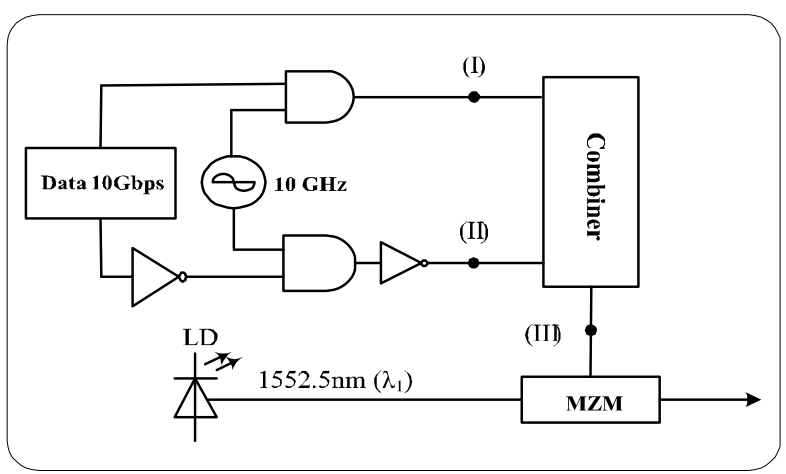

FIG. 1. SCHEMATIC RZ-DPSK TRANSMITTER DESIGN WITHOUT USING ANY PULSE CARVER

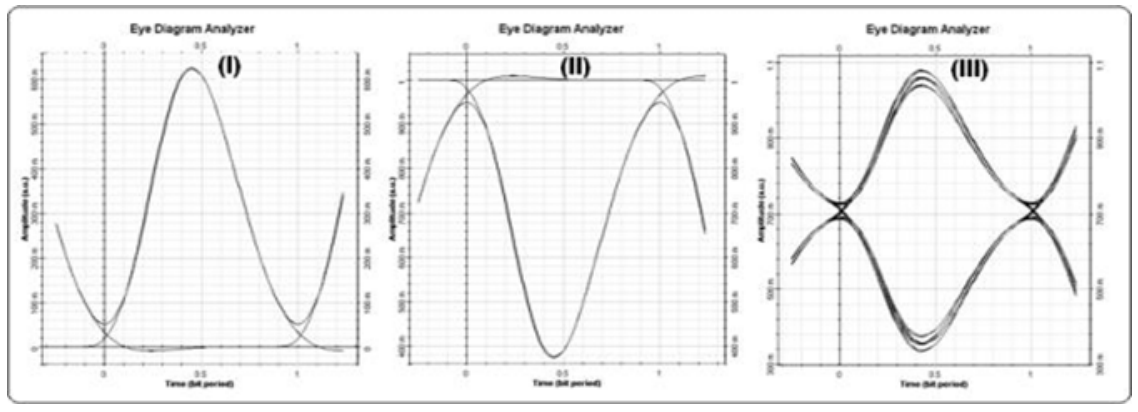

FIG. 2. CORRESPONDING EYE DIAGRAMS AT POINTS (I-III) IN FIG. 1

Mehran University Research Journal of Engineering \& Technology, Volume 36, No. 1, January, 2017 [p-ISSN: 0254-7821, e-ISSN: 2413-7219] 
between adjoining bits. These voltages are applied to operate a single drive $\mathrm{x}$-cut $\mathrm{LiNbO}_{3} \mathrm{Mach}-\mathrm{Zehnder}$ Modulator (LN-MZM), biased at null in a push pull manner, generates a chirp free RZ-DPSK signal. The two states $+1,-1$, are used to produce the two optical phase states separated by " $\pi$ " whereas the " 0 " state is used to create the RZ pulse pattern thus generating RZ-DPSK signal without requiring any pulse carver. The electrooptic transfer function of the MZM biased at null, and the combined optical field at the output of the MZN can be mathematically expressed as:

$E_{\text {output }}(t)=E_{\text {input }}(t)\left(\frac{e^{\frac{i n V_{1}(t)}{V \pi}}-e^{\frac{i n V_{2}(t)}{V \pi}}}{2}\right)$

Where $\mathrm{v}_{1}(\mathrm{t})$ and $\mathrm{v}_{2}(\mathrm{t})$ are the time varying applied voltages on the two arms of the MZM, and $V_{\pi}$ is the voltage needed to introduce an optical phase change of $\pi$-degree on the optical wave passing through one arm of the MZM . As, $v_{2}(t)=-v_{1}(t)$, so the output optical field can be written as:

$\frac{E_{\text {output }}(t)}{E_{\text {input }}(t)}=\sin \left(\pi \frac{\Delta v_{12}(t)}{2 v_{\pi}}\right)$

$\Delta \mathrm{v}_{12}(\mathrm{t})$ is the applied voltages difference between the two arms of the modulator. The optical intensity transfer function is expressed below:

$\left|\frac{E_{\text {output }}(t)}{E_{\text {input }}(t)}\right|^{2}=\sin ^{2}\left(\pi \frac{\Delta v_{12}(t)}{2 v_{\pi}}\right)$

The optical phase across each bit is always identical, i.e. either " 0 " or " $\pi$ ", so the generated RZ-DPSK signal is intrinsically chirp free or have no phase variations across each transmitted bit period. Hence there required no need for a second modulator to be used as a pulse carver so the transmitter design is very simple and cost effective.
Therefore, such novel transmitter design can easily be utilized in a colorless WDM-PON for implementation of an RZ-DPSK uplink with improved transmission budget loss for extended reach as compared to OOK uplink transmission.

\section{SIMULATION MODEL SETUP}

A simulation model is designed in Opti-system software to verify the theoretical analysis for simultaneous transmission of RZ-DPSK data in downlink as well as in uplink respectively. The downlink transmitter can be divided in to two main sections. The first section is "OCSsection" used to generate the optical carrier signals for downlink and uplink whereas the second section known as "modulator section" is used to impress data on the downlink carrier as shown in Fig. 3 (given at the top of page for clarity). Two laser source are used to generate optical wavelengths of $1552.5 \mathrm{~nm}\left(\lambda_{1}\right)$ and $1551.7 \mathrm{~nm}\left(\lambda_{2}\right)$, each having a linewidth of $0.1 \mathrm{MHz}$ with a $12 \mathrm{dBm}$ optical power respectively. Each wavelength is subject to modulate by a $10 \mathrm{GHz}$ sinusoidal RF signal having amplitude of 4V, via an MZM biased at zero, operating over a switching voltage of 4 V.The modulation index " $\mathrm{m}_{\mathrm{h}}$ " is set to 2.405 , results in a complete suppression of the optical carrier whereas the odd-order sidebands are suppressed due to the destructive interference as shown in Fig. 4(a). The output optical spectrum generated thus includes only second order sidebands with a frequency spacing of $40 \mathrm{GHz}$ and $0 \mathrm{dBm}$ power. Due to very small amplitudes $(\leq-20 \mathrm{dBm})$ the other higher even-order sidebands are not observed. An optical filter is used to separate the $2^{\text {nd }}$ order upper sideband from the lower side band and subjected to the modulator section for generating a 10Gbps RZ-DPSK shape data signal as shown in Fig. 4(b-d) respectively. Afterwards an optical coupler is utilized to re-unite the two sidebands before transmitting toward the ONU. 
It can be easily observed from the optical spectrum in Fig. 4(e) that the upper sideband gets phase modulated having $-8 \mathrm{dBm}$ power, while the lower sideband with an optical power level of -4dbm remained blank being unmodulated. Fig. 4(f) is showing the multiplexed optical spectrum of both downlink channels. At the receiving ONU at a distance of $50 \mathrm{~km}$ away from the OLT an optical interleaver is used to separate the two side bands. The upper side band is delivered to the downlink receiver for data retrieval, whereas the empty lower side band signal is subject to the uplink transmitter to be modulated with a $10 \mathrm{Gbps}$ data for the uplink direction as shown in
Fig. 5(g-h). The uplink transmitter configuration includes only the modulator section and function just like used as for the downlink case as shown in Fig. 3. The multiplexed optical spectrum shown in Fig. 5(i) clearly indicates that the uplink data format is also an RZ-DPSK shape signal. Both the downlink and uplink receivers at the ONU and OLT are the conventional MZDI (Mach Zehnder Delay Interferometric) demodulators used to convert the phase-modulated RZ-DPSK signal to an intensity-modulated signal for retrieval of data respectively.

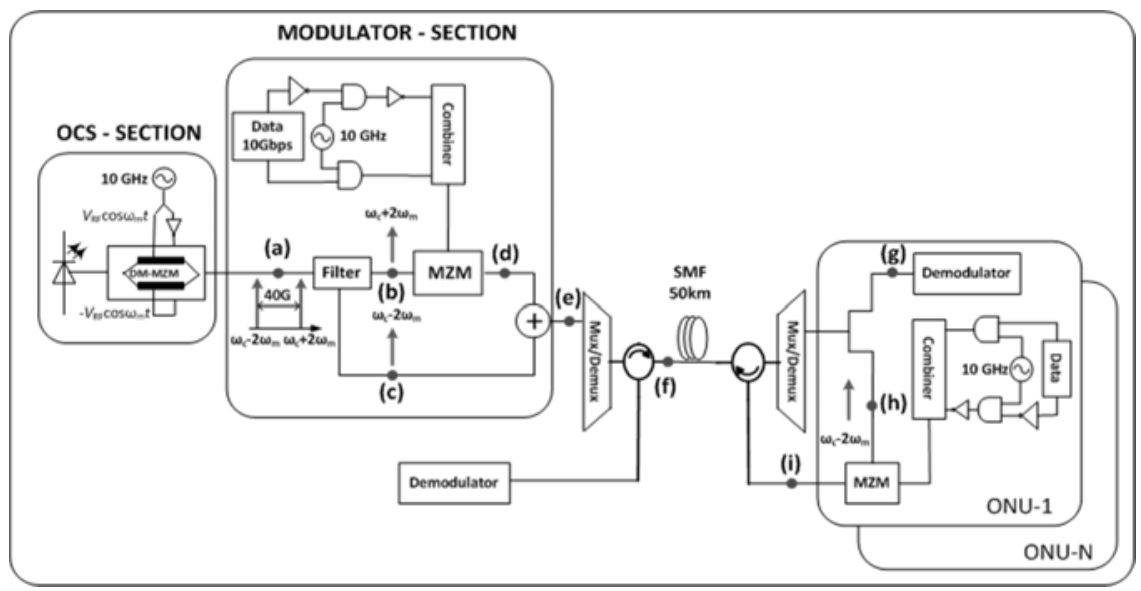

FIG. 3. SCHEMATIC DIAGRAM OF PROPOSED WDM-PON ARCHITECTURE
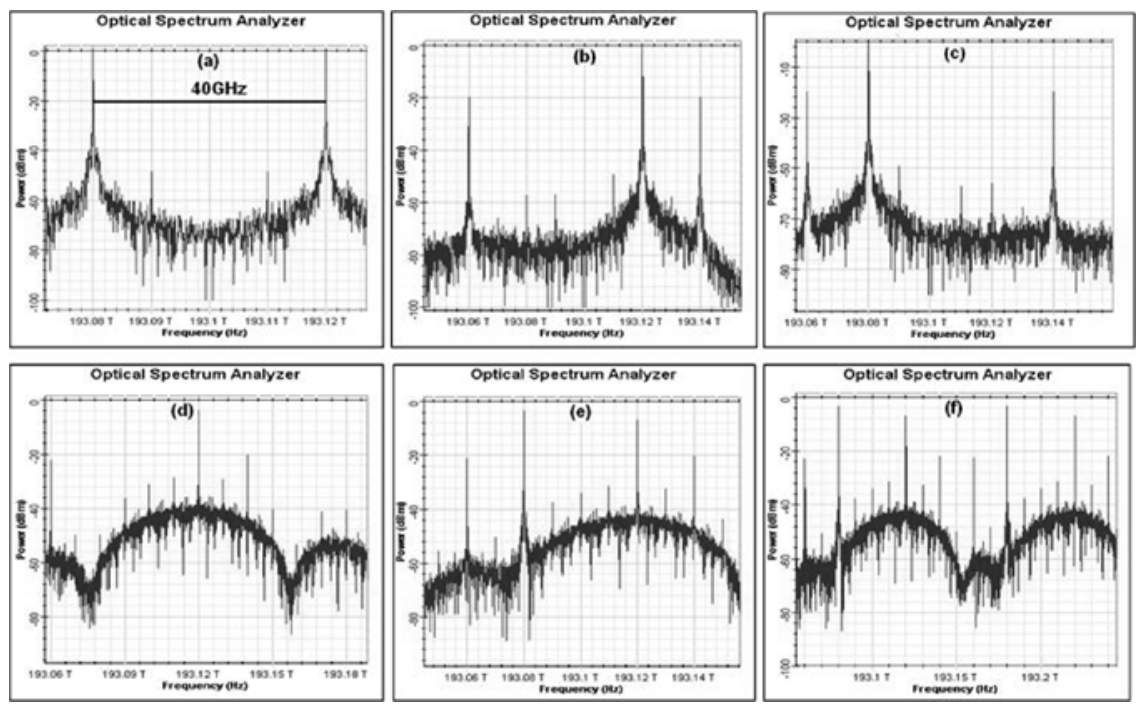

FIG. 4. THE OPTICAL FREQUENCY SPECTRA AT POINTS (A-F) IN FIG. 3

Mehran University Research Journal of Engineering \& Technology, Volume 36, No. 1, January, 2017 [p-ISSN: 0254-7821, e-ISSN: 2413-7219] 
A single feeder fiber configuration is used for a full duplex transmission operation. The different wavelength bands used for downlink and uplink transmission direction along with the combination of AWG and inter-leaver used at ONU help in mitigating RBS and reflection noises effects [17].

\section{PERFORMANCE ANALYSIS}

To validate this novel theoretical scheme, BER (Bit Error Rate) curves depicting a function of received optical power for both RZ-DPSK downstream(B2B and $50 \mathrm{~km}$ ) and RZ-DPSK upstream (100 km) for two channels case are measured as illustrated in Fig. 6.

It is clearly evident from Fig. 6 that the performance of the RZ-DPSK downlink channels is satisfactory with a power penalty of approximately $4.5 \mathrm{~dB}$ for channel 1 and $3.5 \mathrm{~dB}$ for channel 2 over a distance of $50 \mathrm{~km}$ respectively. Similarly, the downlink BER curves show steeper trend as compared to the uplink curves indicating less transmission deterioration over half of the distance as compared to the uplink case as shown in Figs. 6-7. Due to single feeder fiber architecture the B2B BER analysis for the uplink channels cannot be carried out for verification of uplink power penalty. But the overall performance of the uplink channels over a $100 \mathrm{~km}$ round trip distance is seem to satisfactory.

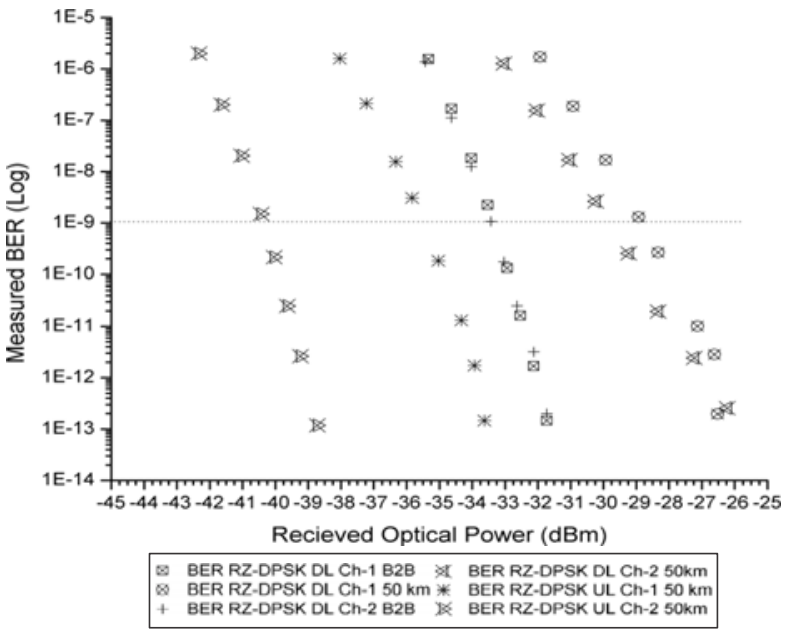

FIG. 6. BER GRAPH FOR RZ-DPSK DL \& UL CHANNELS (50KM AND B2B)

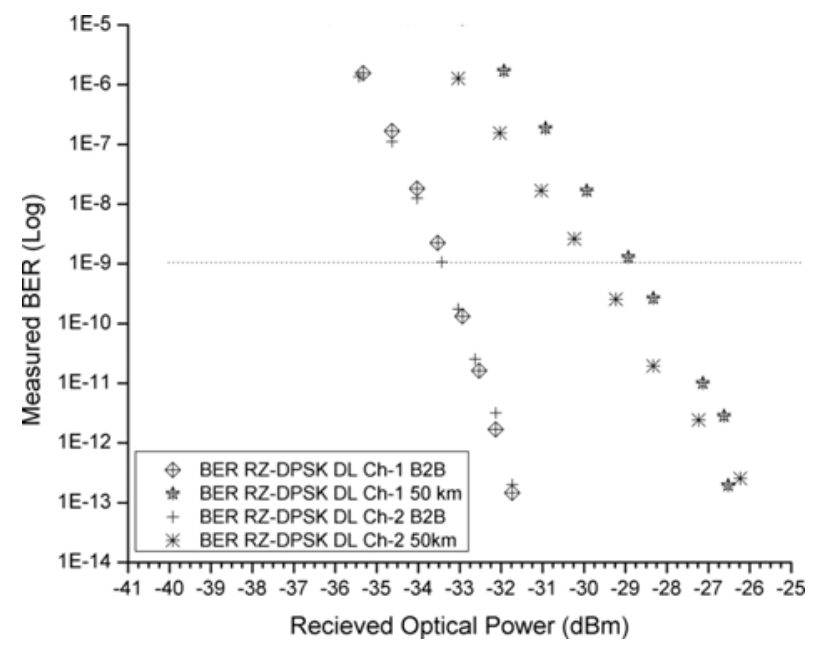

FIG. 7. BER GRAPH FOR RZ-DPSK DL CHANNELS (50KM AND B2B)
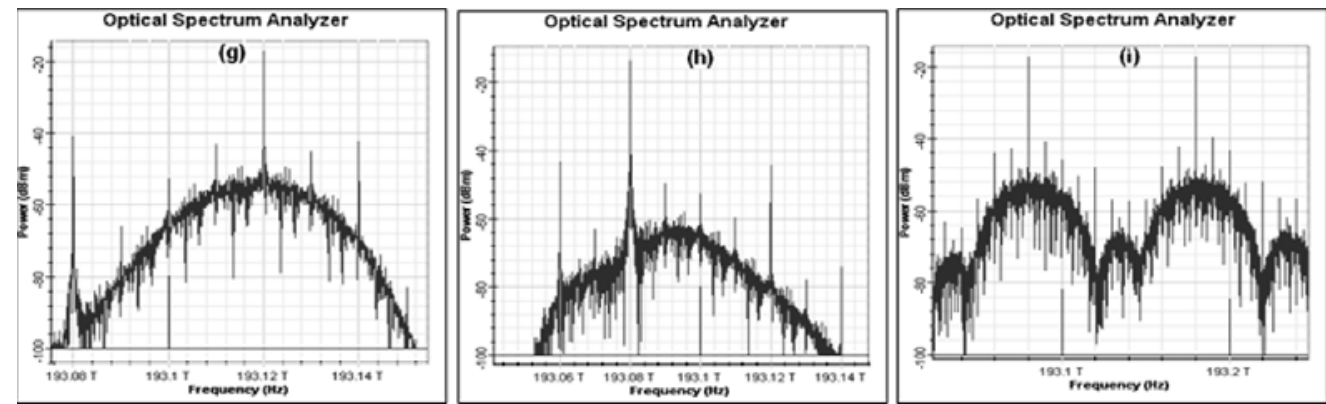

FIG. 5. THE OPTICAL FREQUENCY SPECTRA AT POINTS (G-I) IN FIGS. 1-3

Mehran University Research Journal of Engineering \& Technology, Volume 36, No. 1, January, 2017 [p-ISSN: 0254-7821, e-ISSN: 2413-7219] 
Fig. 8 are shows the corresponding eye diagrams for downlink (B2B and $50 \mathrm{~km})$ and uplink $(100 \mathrm{~km})$ for both multiplexed channels respectively. As a single tone modulation is used therefore, no influence of the bit walkoff phenomenon is observed. Furthermore, in single tone transmission the signal pulses have a constant width and are less or slowly influenced by the fiber dispersion effect which can also be cleared from the eye diagrams.

Fig. 9 gives the eye diagrams of the simulated results. In Fig. 9(a-b), eye diagrams of channel 1 for B2b and $50 \mathrm{~km}$ in the downlink directions are given in Fig. 9(c-d) are for channel 2 in the downlink for B2B and $50 \mathrm{~km}$ respectively. In the last Fig. 9(e-f), eye diagrams for the uplink channel 1 and 2 are shown respectively. The eyes are clear and wide open which signify the applicability of our proposed scheme as easily to implement with reduced inventory, necessitate no bandwidth management for uplink and demonstrated cost effective colorless performance up to $50 \mathrm{~km}$ without requiring the need of any amplification.

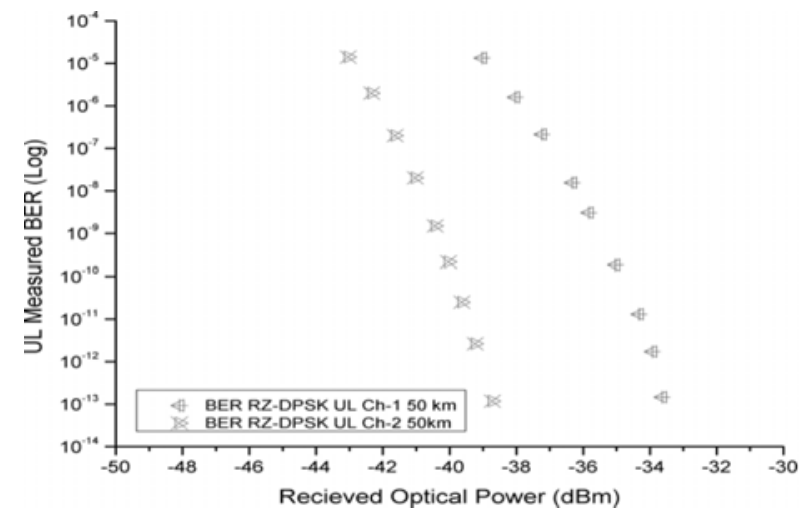

FIG. 8. BER GRAPH FOR RZ-DPSK UL CHANNELS (50KM)
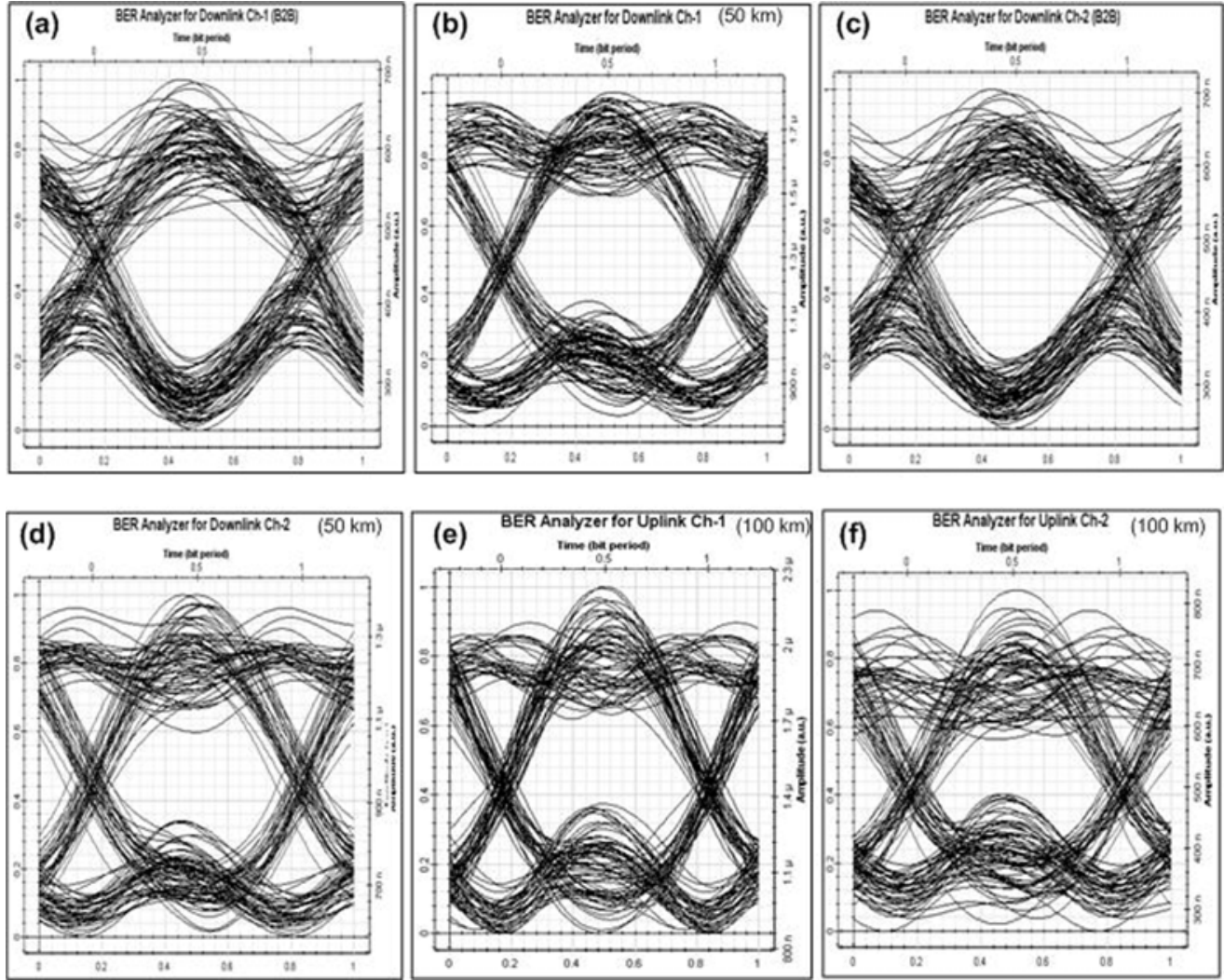

FIG. 9. CORRESPONDING EYE DIAGRAMS FOR BOTH MULTIPLEXED CHANNELS DL AND UL

Mehran University Research Journal of Engineering \& Technology, Volume 36, No. 1, January, 2017 [p-ISSN: 0254-7821, e-ISSN: 2413-7219] 


\section{CONCLUSION}

In this paper a theoretical investigation and generation of simultaneous transmission of a 10Gbps RZ-DPSK data signal for downstream as well as for upstream has been simulated. An OCS scheme for generation of second order dual side-band optical carrier is utilized by quadrupling a $10 \mathrm{GHz}$ clock-frequency with a $10 \mathrm{GHz} \mathrm{LN}$ MZM. The upper side second order band is used to generate an RZ-DPSK data signal at the OLT. At the receiving ONU $50 \mathrm{~km}$ away from the OLT the unmodulated lower side second order band coupled with the downlink transmitted signal is separated for the uplink modulation of $10 \mathrm{Gbps}$ data in RZ-DPSK format. No pulse carver is being used at either transmission end. The simulation results clearly advocate the performance of the single-tone RZ-DPSK data modulation format as a suitable choice for the WDMPON link with a transmission span of $50 \mathrm{~km}$. The proposed architecture eliminated the need of any pulse carver, mid-span power amplifiers along with the requirement of any power splitting device used in the ONU for colorless uplink transmission. Hence the proposed scheme can be claimed as a highly robust, cost effective, backward compatible as well as future proof WDM-PON architecture.

\section{ACKNOWLEDGEMENT}

Authors would like to thank Prof. Xin Xiangjun, Beijing University of Posts \& Telecommunications, University Beijing, China, for his guidance and untiring support.

\section{REFERENCES}

Talli, G., and Townsend, P.D., "Hybrid DWDM-TDM Long-Reach PON for Next-Generation Optical Access", Journal of Lightwave Technology, Volume 24, No. 7, pp. 2827-2834, 2006.

[2]

Lee, S.M., Kim, M.H., and Lee, C.H., "Demonstration of a Bi-Directional 80Km Reach DWDM-PON with 8Gb/s Capacity”, IEEE Photonics Technology Letters, Volume 19, No. 6, pp. 405-408, 2007.
[3] Lazaro, J.A., Prat, J., Chanclou, P.G, Belefû, M.T., Teixeira, A., Tomkos, I., Soila,R., and Koratzinos, V., "Scalable Extended Reach PON", Proceedings of Conference on Optical Fiber Communication/National Fiber Optic Engineers, OThL2, 2008.

[4] Song, H., Kim, B.W., and Mukherjee, B., "Multi-Thread Pollling: A Dynamic Bandwidth Scheme in Long-Reach PON", Journal of Selected Areas in Communications, Volume 27, No. 2, pp. 133-142, 2009.

Suzuki, N., and Nakagawa, J., "First Demonstration of Full Burst Optical Ampliûed GEPON Uplink with Extended System Budget of up to $128 \mathrm{ONU}$ and $58 \mathrm{~km}$ Reach", Proceedings of European Conference on Optical Communication, pp. 141-142, 2005.

[6] Fukada, Y., Nakanishi, T., Suzuki, K.-I., Yoshimoto, N., and Tsubokawa, M., "Gain-Clamp Light Auto Level Control (GCL-ALC) Technique for Gain-Controllable Burst-Mode PON Amplifying Repeater", Proceedings of Conference on Optical Fiber Communication/National Fiber Optic Engineers, OThT5, 2008.

[7] Wong, E., and Lee, K.L., "Automatic Protection, Restoration and Survivability of Long-Reach Passive Optical Networks", Proceedings of IEEE International Conference on Communication, pp. 1-6, 2011.

[8] Zhang, F., Fu, S., Wu, J., Xu, K., Sun, X., Li, W., Li, Y., Hong, X., Lin, J., and Shum, P., "A Wavelength-DivisionMultiplexed Passive Optical Network with Simultaneous Centralized Light Source and Broadcast Capability", IEEE Photonics Journal, Volume 2, No. 3,pp. 445-453, 2010.

[9] Akanbi, O., Yu, J., and Chang, G.-K., "A New Scheme for Bidirectional WDM-PON Using Upstream and Downstream Channels Generated by Optical Carrier Suppression and Separation Technique", Journal of IEEE Photonics Technology Letters, Volume 18, Nos. 1-4, pp. 340-342, 2006.

[10] Fujiwara, M., Kani, J.-I.,Suzuki, H., and Iwatsuki, K., "Impact of Back Reflection on Upstream Transmission in WDM Single-Fiber Loopback Access Networks", Journal of Lightwave Technology, Volume 24, No. 2, pp. 740-746, 2006. 
[11] Hussain, A., Xin, X., Latif, A., Hussain, A., Yu, C.-X., Munir, A., Khan, Y., and Afridi, M.I., "A Novel Symmetric $10 \mathrm{Gbit} / \mathrm{s}$ Architecture with a Single Feeder Fiber for WDM-PON Based on Chirp-Managed Laser", Journal of Optoelectronics Letters, Volume 8, No. 6, pp. 468$472,2012$.

[12] Ferdous, S., Zaman, M.S., Khandaker, F.I., and Majumder, S.P., "Effect of Cross Phase Modulation (XPM) on the Bit Error Rate Performance of an Optical CDMA (OCDMA) System", Proceedings of Conference on Electrical and Computer Engineering, pp. 242-246, 2008 .

[13] Huang, Q., Peng, P.-C., Fu, S.-F., Yang, W.-Y., Huang, J.H., and Yee, H.-H., "Double Side-band with Optical Carrier Suppression Scheme for Broadcasting Transmission", Journal of IEEE Photonics Technology Letters., Volume 26, No. 12, pp. 1172-1175, 2014.

[14] Song, H.-J., Lee, J.-S., and Song, J.-I., "Error-Free Simultaneous All-Optical Up-Conversion of WDM RadioOver-Fber Signals", Journal of IEEE Photonics Technology Letters, Volume17, No. 8, pp. 1731-1733, 2005 .
[15] Yu, J., Gu, J., Liu, X., Jia, Z., and Chang, G.-K., "Seamless Integration of an $8 \times 2.5 \mathrm{~Gb} / \mathrm{s}$ WDM-PON and RadioOver-Fber Using All-Optical Up-Conversion Based on Raman-Assisted FWM", Journal of IEEE Photonics Technology Letters, Volume 17, No. 9, pp. 1986-1988, 2005.

[16] Zhou, M., Ma, J., Yu, C., Xin, X., Huang, H., Rao, L., Zhan, Y., and Liang, H., "Transmission Performance of Quadruple Frequency Optical Millimeter-Wave with Single and Dual Carrier Data Modulations", Proceedings of IEEE Communication Technology, pp. 538-543, 2011.

[17] Chowdhury, A., Chien, H.-C., Huang, M.-F., Hsueh, Y.T., and Chang, G.-K., "Rayleigh Backscattering Noise Eliminated Long Reach Bi-directional WDM-PON with 10-Gb/s DPSK Downstream and Remodulated 2.5-Gb/s OOK Upstream using Optical Carrier-suppressed SubCarrier Modulation", Proceedings of IEEE Lasers and Electro-Optics Society, 2008. 